\title{
An actuarial approach to foreign currency option pricing
}

\section{Zhang Min}

School of Math and Physics, University of South China, Hengyan, 421001 China

Abstract : Using physical probabilistic measure of price process and the principle of fair premium, we deal with pricing formula of option on Foreign currency option under the assumption that foreign option price process driven by fractional Brownian motion process, we obtain the pricing formula of foreign option.

Keywords: fair premium option pricing foreign currency option fractional Brownian motion.

\section{1.introduction}

In 1973 by Professor Black and Professor Scholes, assuming the stock price follows geometric Brown motion, the expected rate of return and volatility of the stock are under constant conditions, access to the famous Black-Scholes options pricing formula[1]. Later many scholars through relaxed Black-Scholes model of some conditions, proposed has various option pricing model, and these traditional of option pricing model roughly is divided into three class: solutions partial differential equation, and discrete model approximation and martingale method, but these method are exists some problem: first its pricing method are is based on no arbitrage balanced complete of market assumed, if market has arbitrage or not completely Shi, equivalent martingale measure on not exists or exists not only, then traditional of pricing method on has difficult; Other Black-Scholes model and, later, a number of scholars have suggested that many of the models have some relationship with the geometric Brown movement, but empirical studies have found that geometric Brown motion was not portrayed the ideal tool for stock prices. Lo and Mackinlary [2], Jackwerth [3] by analysis of measured data geometric Brown motion and market lags, the analysis results showed that with self-similarity of stock price fluctuation, long-term dependency and other features. Geometry has a self-similarity of fractal Brown movement, long term dependency, which enables geometric fractal Brown motion to better characterize the stock price fluctuations. Roger.L.C [4], assuming the stock price follows geometric fractal Brown motion, using the risk-free securities and shares of the class constructs a self financing risk-free arbitrage opportunities, explains the is are arbitrage in financial markets, so the price follows geometric fractal Brown motion options on assets, not pricing by a no-arbitrage pricing theory. In order to solve the above problems many scholars have put forward a lot of solutions in numerous scholar Mogens Bladt,Tina Hviid Rydberg[5] was first raised in 1998, an insurance actuary, which market assumptions in the absence of arbitrage-free, complete and balanced, and price movement of stocks assuming the premise presented precise option pricing formula, and proves that when the stock price follows geometric Brown movement, Actuarial pricing and Black-Scholes pricing agreement. Actuarial basic idea is fair premium principle: the long party buys one stage right, short party will bear a certain potential risks within the validity of the option, 
to that risk with insurance from loss, the premium is the price of this option. This option pricing problem is converted to short party bear the risk of the option terms. Because of the absence of the above assumptions of market economy, so it's effective for general market. This article no such assumption and in financial markets at home and abroad under a risk-free interest rate is constant, actuarial science applied to foreign currency options ...

Foreign currency option is an option for modern financial markets are widely used. Before a specific date in the future, based on the expiration date of the exchange price, Foreign currency options are the right to buy or sell a specific quantity of the Exchange. Income equal to the final maturity date currency in Foreign currency call option prices over finalizing the exchange price value. Currency put options gains equal to finalise prices exceed the expiration date of the exchange price of foreign exchange values. So it's options expiration date $t=T$, earnings $Q(T)-a$ or $a-Q(T)$,

which $Q(t)$ stated that $t$ time closing price of foreign exchange, foreign currency option strike price.

\section{Exchange option insurance fine is}

\section{pricing model}

Considered continuous time of financial markets only two species assets: a is no risk assets, in $t$ moments has no risk rates of no risk assets $r$; another a for risk assets $t$ moments of price, considered time interval $[0, \mathrm{~T}]$, $\{Q(t): t>0\} \quad$ is a defines in a complete

probability space $(\Omega, F, P)$ of random process,
$\left\{F_{t}, t>0\right\}$ is by $Q(t)$ generated of $\sigma-$ algebra, $Q(0)=Q$ is greater than zero of constants

Assuming risk asset price process of foreign exchange for $\{Q(t): t>0\}$, risk-free bond prices for $\left\{B_{t}^{f}: t>0\right\}$ abroad, meet the respective domestic process for $\left\{B_{t}^{d}: t>0\right\}$ risk-free bonds:

$$
\begin{array}{lc}
Q(0)=Q & d Q(t)=Q(t) \mu d(t)+Q(t) \delta d W(t) \\
B_{T}^{f}=1 & B \stackrel{f}{=} e^{-f_{r}(T-t)}=B_{t}^{f} r^{f} d t \\
B_{T}^{d}=1 & B \stackrel{d}{=} e^{-d_{r}^{d}(T-t)} d B_{t}^{d}=B_{t}^{d} r^{d} d t
\end{array}
$$

Among them $\mu, \delta$ is a negative constant, yields for Foreign currency options, price fluctuations of foreign exchange rates is a constant risk-free interest rate at home and abroad, respectively, for standard Brownian motion on a probability space, and the algebra generated by (the flow).

Definitions 2.1: Assumptions $\beta(t)$ for the $Q(t)$ instantaneous rate of real-valued functions of time, Foreign currency option prices $Q(t)$ produced in the process and expects yields $\int_{0}^{t} \beta(s) d s$ to meet $\frac{E Q(t)}{Q}=e^{\int_{0}^{t} \beta(s) d s}$.

$C(a, T)$ and $P(a, T)$ are the $Q(t)$ represent the foreign exchange price, $a$ for strike price, 
expiration date $T$ for the actuarial pricing Foreign currency option call and put options.

Defines 2.2: At $t=0$,European currency options with no paid bonus by Actuarial pricing is :

$$
C(a, T)=E\left(\left(e^{-\int_{0}^{T} \beta(t) d t} Q(T) e^{-r^{f} T} B_{T}^{f}-a e^{-r^{d} T} B_{T}^{d}\right) I_{A}\right)
$$

$P(a, T)=E\left(\left(a e^{-r^{d} T} B_{T}^{d}-e^{-\int_{0}^{T} \beta(t) d t} Q(T) e^{-r^{f} T} B_{T}^{f}\right) I_{B}\right)$

which $I_{A}$ and $I_{b}$ respectively are $A$ and

$B$ function, to be executed options at the maturity date of the conditions is

conditions a $e^{-\int_{0}^{T} \beta(t) d t} Q(T) e^{-r^{f} T} B_{T}^{f}>a e^{-r^{d} T} B_{T}^{d}$

conditions b $\quad a e^{-r^{d} T} B_{T}^{d}>e^{-\int_{0}^{T} \beta(t) d t} Q(T) e^{-r^{f} T} B_{T}^{f}$

Note: (1)Defines 2.2 has no any assumed on financial markets, its results are no effective for no arbitrage balanced of complete market. In calculating the potential risk of loss only risk asset according to the expected rate of return of discounted, the risk-free asset discounted at the risk-free interest rate.

(2)Different from the traditional conditions $Q(T) e^{-r^{f} T} B_{T}^{f}>a B_{T}^{d} \quad, \quad$ actuarial models executable condition is $e^{-\int^{T} \beta(t) d t} Q(T) e^{-r^{f} T} B_{T}^{f}>a e^{-r^{d} T} B_{T}^{d}$.
(3) the price process without any restrictions in the definition, only needs to know the actual probability distribution to calculate option prices.

\section{Currency options market model}

Foreign currency option market model 1968 Mandelbrot Van Ness[6] was first introduced to geometric fractal Brown motion , it is mainly used to describe the nature of irregular shapes such as mountain ranges, coastlines, terrain and simulate various fractal characteristic of noise tracks and so on, is now a mathematical models of random processes. Statistical analysis of stock price changes in stock prices will be discovered when there is a good rule, Mandelbrot had found that stock price changes per unit time distribution follows a certain fractal dimension of symmetric stable distributions; regardless of how much per unit time, its distribution is similar, namely, change scale can be equally distributed as appropriate. From Visual Shang can will points shaped of features antibody for: since similar sex, and no features length, and has fine structure or local to a species way and overall similar, these is most shares behavior are has of features, so with points shaped geometry Brown movement process to description stock price process to than geometry Brown movement more fine more accurate, can said points shaped Brown is stock price behavior of height realistic.

Foreign currency prices swings have similar stock price fluctuations, so consider Foreign currency fractal price follows geometric Brown movement. Intuitive geometric fractal Brown motion parameter $H$ is used to indicate the stock price on the roughness of the graphic, $H$ from 1 to 0 , geometrical fractal 
Brown movement structures become more sophisticated, and branches of larger dimension graphics are becoming more unusual, so we only take into account foreign exchange price follows geometric fractal Brown motion parameters $H(1 / 2<H<1)$.

Definitions 3.1 if the center of Gaussian processes $B_{H}(t)=\left\{B_{H}(t), t>0\right\}$ with covariance function has the following form:

$E\left(B_{H}(s), B_{H}(t)\right)=\frac{V_{H}}{2}\left(s^{2 H}+t^{2 H}-|t-s|^{2 H}\right)$

The constant $\quad V_{H}$ for standardization, $\quad V_{H}=\frac{\Gamma(2-2 H) \cos (H \pi)}{\pi H(1-2 H)} \quad$ and $B_{H}(0)=0$, we call the geometrical fractal Brown movement of $B_{H}=\left\{B_{H}(t), t \geq 0\right\}$ with $H \in(0,1)$

parameter,clearly

$$
B_{H}(t) \sim N\left(0, V_{H} t^{2 H}\right) .
$$

Lemma 3.2 geometric Brown motion satisfies the following differential equation[7]:

$$
d S_{t}=\mu S_{t} d t+\sigma S_{t} d B_{t}
$$

$$
\mu \in R, \sigma>0, S_{0}=S \quad t \in[0, T]
$$

$$
\begin{aligned}
& \text { has } \begin{array}{l}
\text { a unique } \\
S_{t}=S \exp \left\{\left(\mu-\frac{1}{2} \sigma^{2}\right) t+\sigma B_{t}\right\}
\end{array} \\
& \text { geometric fractal Brown motion } B_{H}(t)
\end{aligned}
$$

instead of $B_{t}$ the stochastic differential equations (4),

$$
\begin{array}{r}
d S_{t}=\mu S_{t} d t+\sigma S_{t} d B_{H} \\
\mu \in R, \sigma>0, S_{0}=S \quad t \in[0, T]
\end{array}
$$

For $H \neq \frac{1}{2}, \quad B_{H}(t)$ is Not half a martingale, $\mathrm{SDE}(5)$ cannot use formulas Itô to solve the equations. At that time, Dasgupta [8] proved by Riemann sum converges to the following conclusion:

Lemma 3.3 when $\frac{1}{2}<H<1$, the SDE

solution has similar geometric Brown motion solutions

$$
S_{t}=S \exp \left\{\mu t+\sigma B_{H}(t)\right\}
$$

The parameter for the $\mathrm{H}, B_{H}(t) \sim N\left(0, V_{H} t^{2 H}\right)$

By the lemma available

$$
E \Phi)=S \exp \left(u t+\frac{\sigma^{2} V_{H} t^{2 H}}{2}\right)
$$

Theorem 3.4 a foreign currency price process meet $S D E(5)$, and

$$
\begin{aligned}
& C(a, T)=B_{0}^{f} Q N\left(d_{1}\right)-a B_{0}^{d} N\left(d_{2}\right) \\
& P(a, T)=a B_{0}^{d} N\left(-d_{2}\right)-B_{0}^{f} Q N\left(-d_{1}\right)
\end{aligned}
$$

In the above formula $\quad d_{1}=d_{2}+\sigma \sqrt{V_{H}} T^{H}$

$$
\begin{gathered}
d_{2}=\frac{\ln \frac{B_{0}^{f} Q}{B_{0}^{d} a}+r^{d} T-\frac{1}{2} \sigma^{2} V_{H} T^{2 H}}{\sigma \sqrt{V_{H}} T^{H}} \\
N(x)=\int_{-\infty}^{x} \frac{1}{\sqrt{2 \pi}} e^{-\frac{y^{2}}{2}} d y=P(\zeta<x)
\end{gathered}
$$

其中 $\zeta \sim N(0,1)$ 
Proof: defined by lemma 3.3 and 3.1

$$
\begin{gathered}
\exp \left\{\int_{0}^{T} \beta(t) d t\right\}=\exp \left(\mu T+\frac{\sigma^{2} V_{H} T^{2 H}}{2}\right) \\
\ln \frac{B_{T}^{f} Q(T)}{B_{0}^{f} Q} \sim N\left(\mu T, \sigma^{2} V_{H} T^{2 H}\right) \\
\quad \int_{0}^{T} \beta(t) d t{ }_{0}^{f} B Q T>B_{j}^{d} a
\end{gathered}
$$

Call options execution condition $A$

So

$$
\begin{gathered}
-\left(\mu T+\frac{1}{2} \sigma^{2} v_{H} T^{2 H}\right)+\ln B_{0}^{f} Q+\left(\mu T+\sigma B_{H}(T)\right)>\ln B_{0}^{d} a \\
B_{H}(T)>\frac{\ln \frac{B_{0}^{d} a}{B_{0}^{f} Q}+\frac{1}{2} \sigma^{2} V_{H} T^{2 H}}{\sigma} \\
y=\frac{\ln \frac{B_{0}^{f} a}{B_{o}^{f} Q}+\frac{1}{2} \sigma^{2} V_{H} T^{H}}{\sigma} \\
C(a, T)=E\left(\left(e^{-\int_{0}^{T} \beta(t) d t} e^{-r^{f} T} B_{T}^{f} Q(T)-e^{-r^{d} T} B_{T}^{d} a\right) I_{C}\right) \\
\left.E_{0}^{-\int_{0}^{T} \beta(t) d t} e^{-r^{f} T} B_{T}^{f} Q(T) I_{C}\right)-E\left(e^{-r^{d} T} B_{T}^{d} a I_{C}\right) \\
=I_{1}-I_{2}
\end{gathered}
$$$$
I_{1}=E\left(\exp \left\{-\int_{0}^{T} \beta(t) d t\right\} e^{-r^{f} T} B_{T}^{f} Q(T) I_{C}\right)=E\left(B_{0}^{f} Q \exp \left\{\sigma B_{H}(T)-\frac{1}{2} \sigma^{2} V_{H} T^{2 H}\right\} I_{C}\right)
$$$$
=B_{0}^{f} Q \int_{y}^{\infty} \frac{1}{\sqrt{2 \pi V_{H}} T^{H}} e^{-\frac{\left(x-V_{H} T^{2 H} \sigma\right)^{2}}{2 V_{H} T^{2 H}}} d x
$$$$
=B_{0}^{f} Q P\{Z>y\} \quad \text { 其中 } Z \sim N\left(V_{H} T^{2 H} \sigma, V_{H} T^{2 H}\right)
$$$$
=B_{0}^{f} Q P\left\{\frac{\left.Z-V_{H} T^{2 H} \sigma\right)}{\sqrt{V_{H}} T^{H}}<\frac{y-V_{H} T^{2 H} \sigma}{\sqrt{V_{H}} T^{H}}\right\}
$$ 


$$
\begin{aligned}
& =B_{0}^{f} Q P\left\{\frac{Z-V_{H} T^{2 H} \sigma}{\sqrt{V_{H}} T^{H}}<\frac{\ln \frac{B_{0}^{f} Q}{B_{0}^{d} a}-\frac{1}{2} \sigma^{2} V_{H} T^{2 H}}{\sigma \sqrt{V_{H}} T^{H}}+\sigma \sqrt{V_{H}} T^{H}\right) \\
& =B_{0}^{f} Q N\left(d_{1}\right)
\end{aligned}
$$

In the same way we can obtain

$$
\begin{gathered}
I_{2}=E\left(e^{-r^{d} T} B_{0}^{d} a I_{C}\right)=e^{-r^{d}} B_{0}^{d} a N\left(\frac{\ln \frac{B_{0}^{f} Q}{B_{0}^{d} a}-\frac{1}{2} \sigma^{2} V_{H} T^{2 H}}{\sigma \sqrt{V_{H}} T^{H}}\right) \\
=e^{-r^{d} T} B_{0}^{d} a N\left(d_{2}\right) \\
\text { Let } \quad d_{2}=\frac{\ln \frac{B_{0}^{f} Q}{B_{0}^{d} a}-\frac{1}{2} \sigma^{2} V_{H} T^{2 H}}{\sigma \sqrt{V_{H}} T^{H}} \quad d_{1}=d_{2}+\sigma \sqrt{V_{H}} T^{H}
\end{gathered}
$$

therefore

$$
C(a, T)=B_{0}^{f} Q N\left(d_{1}\right)-a B_{0}^{d} N\left(d_{2}\right) .
$$

Results of this theorem and reference [9] in interest rate determined under the Exchange's pricing formula is the same. Indicating that actuarial methods can be applied to foreign currency option pricing, a European foreign currency options for payment of dividends is also similar to get the pricing formula. Empathy can prove sadly.

Note (1) Theorem 3.4 does not appear in the expected rate of return, it shows that no matter how much the expected rate of return does not affect the options pricing. (2) Theorem 3.4 very similar to the Black-Scholes formula, but there is an essential distinction: Brown is a martingale in the Black-Scholes model geometry and geometric fractal Brown Theorem 3.4 is not a semimartingale, he portrays is are arbitrage in the market. When price follows geometric fractal Brown movement, market arbitrage, can't use the no-arbitrage theory of call and put options are given parity.

Corollary 3.5 set prices satisfy the SDE (5) and the present value of the dividend payment, foreign exchange option pricing formula is

$$
C(a, T)=\left(B_{0}^{f} Q-W\right) N\left(d_{1}^{\prime}\right)-a B_{0}^{d} N\left(d_{2}^{\prime}\right)
$$

$$
P(a, T)=a B_{0}^{d} N\left(-d_{2}^{\prime}\right)-\left(B_{0}^{f} Q-W\right) N\left(-d_{1}^{\prime}\right)
$$

In the formula $d_{1}^{\prime}=d_{2}^{\prime}+\sigma \sqrt{V_{H}} T^{H}$

$$
d_{2}^{\prime}=\frac{\ln \frac{B_{0}^{f} Q-W}{B_{0}^{d} a}-\frac{1}{2} \sigma^{2} V_{H} T^{2 H}}{\sigma \sqrt{V_{H}} T^{H}}
$$




\section{CONCLUSIONS}

Literature [4] introduction of option pricing insurance actuary, and proved as the stock price follows geometric Brown movement insurance actuary with the Black-Scholes option pricing formula for arbitrage-free agreement. In this paper we extend this result, assuming the Exchange prices move according to the parameters of geometric fractal Brown movement, proves insurance actuary in reference [9] pricing agreement. Price follows geometric description of the fractal Brown motion can be a good price, but at the moment the market is are arbitrage, so cannot be free, balanced traditional thinking to option pricing. Insurance actuary well avoided the market assumes that the condition, in the absence of any market assumptions, only know that physical probability measure of the price can be accurately priced. Discussed in this paper follow the parameters of foreign exchange is a geometric fractal Brown movement, have similar solutions of geometric Brown motion, resulting in bullish and bearish foreign exchange option pricing formula, and parameters for geometric fractal Brown motion case remains to be further studied and discussed.

\section{Reference}

[1] Black F,Scholes M. The Pricing of option and corporate liabilities[J] Journal of Political Economy, 1973 .81(3):637-659

[2] Andrew W. Lo and A. Craig MacKinlay. Stock market prices do not follow random walks: evidence from a simple specification

test[J].The Society for Financial Studies.Vo 1. 1, No. 1 (Spring, 1988), pp. 41-66

[3] Jens Carsten Jackwerth.Recovering Risk Aversion from Option Prices and Realized Returns[J].J. OF FINANCE, Vol. 51 No. 5, December 1996

[4] Scott, L. Pricing Stock Options in a Jump-Diffusion Model with Stochastic Volatility and Interest Rates: Applications of Fourier Inversion Methods [J]. Mathematical Finance, 1997, 7(4): 413-426.

[5] Bladt M,Rydberg $T$ H.An actuarial approach to option pricing under the physical measure and without market assumptions[J].Insurance:Mathematics and Economics.1998.22(1):65-73

[6] Mandelbrot BB, Van Ness JW (1968) Fractional brownian motions, fractional noises and applications. SIAM Rev.10:, $422-437$

[7] John hull. Options, futures and derivative securities $[\mathrm{M}]$. Zhang TaoWeiYi Beijing: huaxia publishing house1997

[8] A. Dasgupta and G. Kallianpur: "Multiple fractional integrals", Probab. Th. Rel. Fields, Vol. 115, (1999), pp. 527-548.

[9] Musiela M,Rutkowski M.Martingale methods in financial modeling[M].Berlin; Heidelberg; NewYork; Springer Verlag,1997.109-181.

[10] LiShang Jiang . The mathematical model of option pricing, and methods[M] Beijing: higher education press 2003. 